\title{
PERFORMING GOOD TEACHING: THE FRONTSTAGE AND BACKSTAGE WORK OF INTERDISCIPLINARY WORKING
}

\author{
D. Gurbutt, K. Williams \\ University of Central Lancashire (UK)
}

\begin{abstract}
The UK higher education research excellence framework and teaching excellence framework both recognise Interdisciplinary working as a means of helping students prepare for employment and understand a broader range of approaches to problem solving. This has been accompanied by a drive to improve accessibility leading to increased use of flexible learning. The change to students funding their own education via tuition fees has led to higher learner expectations of the student experience.
\end{abstract}

This paper explores the interdisciplinary learning that takes place between staff when they work together to create and perform good teaching and the impact on learners when they begin to recognise and appreciate the extent of the 'team behind good teaching.' Based loosely on Goffman's work on 'Identity' and the concepts of 'frontstage and backstage' work, the paper will explore the shift in culture to using technology enhanced learning approaches to enable staff to engage students in learning, maximise time in the classroom and create higher quality learning products to support collective and personalised learning. The paper will explore the tensions around understandings of learning and teaching and what it means to be a 'good teacher' arguing that increasingly teaching is collaborative by design (if not delivery) and the result of interdisciplinary skill sharing amongst staff working in learning technology and educator roles. This teamwork approach to learning is shifting the culture of approaches to learning to enable increased creativity and responsiveness to student needs.

Keywords: Technology enhanced learning (TEL); student experience; curriculum design.

\section{INTRODUCTION}

Interprofessional Education (IPE) can be defined as "as occasions when two or more professionals learn with, from and about each other to improve collaboration and the quality of care" Centre for Advancement of Interprofessional Education, CAIPE (2017) [1] Interdisciplinary education is a requirement of the professional statutory bodies in Health, Medicine and Social Care in the UK and finding ways to incorporate this type of learning into the curriculum is an important yet sometimes problematic feature of these programmes due to the constraints of timetables and curriculum. Some of the programmes enrol large numbers of students, such as the pre-registration nursing and pharmacy programmes, and some are relatively small, such as the medical sciences programmes which may have cohorts of just 40 students. Designing learning which enables large numbers of students, and uneven numbers of students from different disciplines to work together, presents a particular set of creative challenges. IPE is only a part of the Interdisciplinary Education (IDE) which is undertaken across the university; and all Faculties have examples of collaborative work with students from different disciplines, subjects and backgrounds covering interdisciplinary learning in subjects as diverse as Law and Graphic Design. This collaborative working unleashes a new wave of creativity as students share meaningful learning, but staff also learn from one another. Interdisciplinary education has been one of the ways in which learning and teaching is cross-fertilised throughout the university and supports innovation. Early evaluations have shown that staff learn from each other as a consequence of collaborative student learning. The move towards collaborative and interdisciplinary learning is impacted by internal and external drivers within the sector and the university. This paper seeks to explore and evaluate some of these elements and the synergy between them which is driving a culture change.

One of the key factors in changing the culture of teaching is the development of closer working relationships between the different teams which support teaching. This sometimes includes the recognition that, increasingly, innovative and creative teaching and a positive student experience can be the product of co-production of learning with different educational specialists. This includes learning technologists who can be a key part of the team which delivers good teaching and shapes the learning experience even though technologist and student may not meet in person during a course. To assist in 
conceptualising this, we have drawn on the work of Erving Goffman (1959) [2] who uses the concepts of 'frontstage and backstage' work in the depiction of how we present ourselves in everyday life, using analogies from the world of the theatre. This description highlights the interplay between the 'seen' or visible elements of identity and those which remain hidden. This analogy can usefully be applied to the context of teaching. In the delivery of contemporary teaching there is often a 'performer'; the person who delivers the lecture or teaching episode and the 'performance' which may rely on technical support; as compared with backstage work in the theatre which requires the support of lighting, sound, direction and production. The actor or teacher may be perceived as the 'performer' in this context, but the 'performance' is the result of an interconnected and inter-reliant team who bring the production to life. It is this analogy which describes the interplay which occurs between the 'teaching in the moment' which is the teaching session, and the production and packaging of materials which assist this process. There is also recognition that increasingly some 'teaching 'may never even be 'delivered' in a traditional format by the teacher or tutor. These may form part of the flipped classroom provision or other learning elements which are collaboratively produced and may be accessed by the student in a self-directed 'free range' way of learning. Reflecting on this process of change in learning, the Interdisciplinary learning programmes are used as a vehicle to explore changing patterns of learning. Therefore, in considering culture it is useful to consider: i) how interdisciplinary learning is supported by technology, ii) how interdisciplinary learning takes place between staff - particularly teaching teams and learning technology teams and iii) how cultural shifts in learning can be driven by Centres for Excellence in Learning and Teaching and Technology Enabled Learning Teams.

\section{CONTEXT}

The context of Higher Education in the UK has changed significantly in recent years. The Dearing Report (1997) [3] recommended the introduction of student tuition fees paid by the student to the institution providing higher education. This has led to changes in the ways in which students view their studies as they shifted from being recipients of Higher Education to consumers who make direct payments in exchange for educational provision. The reconfiguration of this relationship has altered the ways in which students see universities and also the ways in which universities view students. In 2012 the university fees tripled in one year to a level which has remained relatively constant in the intervening years. This escalation in costs payable by the student, generated a consumerist approach to education and an increasing focus on the student experience and in particular student satisfaction with programmes. This has led to innovations in the sector focused on trying to determine and evaluate criteria by which a 'good' student experience can be measured, such as the introduction of the National Student Survey in 2005 which follows an annual cycle (HEFCE, 2017) [4] and specific research into specific areas such as student contact hours (NUS, 2012) [5].

There are also other drivers which have accompanied a cultural shift in Higher Education, these include the introduction of the Research Excellence Framework (HEFCE, 2017) [6] and the Teaching Excellence Framework (GovUK, 2017) [7] both of which encourage interdisciplinary working as part of the matrix criteria, and work on the importance of Employability and Enterprise strands in preparing students for the workplace on graduation (Butcher et al , 2011) [8] of which 'real world' experiences which can include IDE can be seen to be a constituent part.

Working in interdisciplinary ways can be a challenge for large universities with multiple and diverse programmes and large numbers of students. Interdisciplinary working has developed in a number of ways, firstly in a grassroots, 'bottom-up' approach from individual staff and teams with an interest in working collaboratively and collectively who identify synergy in teaching areas and identify sessions which can be delivered in interdisciplinary ways or as shared teaching; secondly through developing a horizontal approach to IDE which may be a result of Interprofessional education, e.g. as prescribed by the statutory professional bodies in Health and Social care which places a requirement on course providers to demonstrate where pre-registration practitioners learn together in preparation for professional practice and where this is aligned to the required outcomes for graduates (see GMC , 2015) [9]; thirdly, Interdisciplinary education is driven by the belief that students benefit from learning together and that IDE is supported by strong pedagogical approaches. There is acknowledgement of the benefits of Interdisciplinary learning including 'the ability to change perspectives, to synthesize knowledge of different disciplines, and to cope with complexity.' Spelt et al (2009) [10]. 


\section{CULTURE}

In order to identify how interdisciplinary learning might be extended and consolidated across the university, the Centre for Excellence in Learning and Teaching (CELT) sought to explore how a newly created programme of interdisciplinary education could be enhanced and supported by use of technology and how technology could be used to overcome some of the practical challenges of working with large cohorts of different disciplines.

The creation of a new medical school at the university created the requirement to develop and deliver appropriate interdisciplinary and meaningful learning across large cohorts. There were some particular challenges to consider. Initially all of the medical students on the programme were international students and had limited knowledge of the UK healthcare systems and in order to create meaningful learning for all participants a way had to be found to address this element of learning. Working with learning technologists and eLearning facilitators, the leading tutor identified a software package that would be suitable for flipped learning. Flipped classrooms involve providing material in advance of the session to work through at their own pace in preparation for the classroom session which follows. Students are encouraged to attend the sessions with questions from what they have learned and a level of knowledge which enables them to engage in discussion and apply their knowledge. The tutor sought the advice of learning technologists to identify a package which would suit the context and provide an interactive learning experience which would be readily accessible. Consideration was made of the needs of the end user, the student, but also the content writer, the tutor, who needed a package which would allow material to be created and also regularly updated as policies change. 'Articulate storyline' was chosen for a number of reasons. These included the visual nature of the learning packages produced this way; the software uses 'characters' and 'settings' to take the student through learning narratives. The software requires students to be active participants, the learner needs to advance the 'conversation' between the characters. The software also enables the embedding of video, papers and hyperlinks to learning materials, such as national policies. This approach enabled speakers of English as a second language the opportunity to engage with 'bite sized' chunks of learning at a pace determined by them. This flipped classroom approach enabled students to learn at their own pace and to revisit materials multiple times if necessary, in order to engage with content in a meaningful way in advance of taught sessions (Gurbutt and Gurbutt, 2015) [11].

The flipped classroom elements were developed in conjunction with specialists in use of the software. The software has since been utilised by other tutors across the institution following successful use on this programme. The approach enabled specific learning to be delivered to students via self-directed learning, students were able to engage with core materials required for the session, but also additional materials relating to context if the topics were unfamiliar. Materials included both audio, visual and written elements. These were used not only to deliver content, but also to gain 'added value' for example, some of the audio used local speakers, so that medical students could listen to the accents of local people and attune their ear to local pronunciation, which would prove useful on later placements. The large cohort sessions were centred on an actor-led interactive forum sessions with small group breakout sessions. The scenarios which were used were filmed by technicians and made available to students to revisit following the sessions. Lecture capture technology enables students to access information again, but also enables tutors from different disciplines to engage with material from a different perspective in their own teaching, selecting examples delivered by subject specialists to support their own discussions with student groups.

This experience of using software and lecture capture led to a better understanding of how interdisciplinary learning takes place between staff. There was evidence from evaluations which were conducted following each session and debriefing sessions, that staff as well as students felt that they were learning from each other. This included observing and using different teaching approaches as well as extending their understanding of content and context relating to different professional groups.

Staff comments included: 'I learned something about how other professions see the world from talking to colleagues about a particular issue' and 'this was a good opportunity to reflect on how we might address the same issue in different ways. But interestingly this also applied to learning and teaching: 'I learned a lot from colleagues from classroom management to some useful activities which I will try with my own students' and 'it was really useful to see how different disciplines teach or explain things, I hadn't experienced this since my initial teacher training.' There were also comments on the use of technology enhanced learning: 'it made me want to try new approaches and I felt that there were people around to offer some support' and 'using the various resources made me feel more adventurous in what I feel I might try.' 
This was a welcome way of cross-fertilising teaching approaches and proved to be more engaging for staff than more formal peer observation initiatives. However, apart from teaching staff there was another group of staff which identified the benefits of peer learning. The drivers for flipped classroom and lecture capture, in trying to engage diverse groups of students with teaching materials, highlighted the benefits of educationalists working more closely together, particularly partnerships between classroom educators and learning technologists. Indeed, staff using flipped learning materials readily shared the technology enabled approaches they had utilised themselves, but also the contact details for the learning technologists who had helped them to develop materials.

The highlighting of the broader collaborations between learning technologists and classroom educators was also a result of a university-wide initiative known as 'Digital Shift' this was a service-led project to encourage the wider use of technology in teaching. The university invested widely in technology for classrooms and Surface Pro devices for staff, but also in the training and support of staff in using technology. The work in IDE served as one example to illustrate the effectiveness of this strategy. A project within CELT further highlighted not only the growing extent of interdisciplinary work across the university, but the role played by the learning technology teams in facilitating and supporting many teaching initiatives, not only in developing competence in staff groups, but also in developing confidence.

The university has consolidated this approach in the Learning and Teaching Strategy (2017) [12] which focuses on Pedagogy, Learning Environment, Accessibility, Curriculum and Employability and Enterprise (PLACE). The CELT and the Technology Enabled Learning Team (TELT) underpin all of these strategic areas. The evaluations from the IDE and IPE learning events illustrate clearly how cultural shifts in teaching and learning can be supported by closely allied working between CELT and TELT. Developments have included regular meetings between representatives of the two teams, a TELT skills-based conference which wraps around the university Learning and Teaching Conference enabling staff to learn the techniques that others have described and evaluated in pedagogic papers at the conference and to gain support for their own learning as well as identify colleagues with whom they might be able to collaborate. This Digital Learning Conference focused closely on the exploration and implementation of technologies across academic disciplines. The event comprised of a number of breakout sessions, delivered by academic users. The TELT team operated as facilitators to support the development and transfer of digital approaches across Schools and disciplines. This approach to shared practice offers significant opportunities to consider and discuss the pedagogy with the academic development team ensuring that sound pedagogy drives the learning experience. Working closely with CELT the two teams work alongside each other simultaneously evaluating the range of available technologies best suited to fulfil user requirements and expectations and enhance the student learning experience in the most effective and accessible way.

Involvement of academics and technologist in planning and co-production is encouraged at various stages in the design and delivery of curricula, and recognising the importance of the learning technologist is essential to the successful integration of digital approaches.

The technologies which are utilised across the institution in learning and teaching can be categorised using three themes: content and resources, assessment and feedback, community and collaboration. Working with the academic team the technologists are able to identify the most suitable technologies for a particular programme. These are then discussed and demonstrated to ensure the right tool is used to achieve the learning outcomes of the unit.

Apart from these large annual conferences there is also a programme of smaller conferences and workshops around core themes. A mini conference on 'Cultivating Collaboration' focused on the skills acquisition for using a range of applications which facilitate joint working and exploration of tools which can be used to enable teams to work collaboratively. Some of these will be tools which enable staff to plan sessions with shared development areas, whilst others support co-creation and delivery options which allow for the specific needs of particular disciplines to be met alongside core components. This ability to work flexibly and accessibly in planning and review of materials is important as evaluations with colleagues indicate that timetable constraints and being able to regularly meet in person with other staff to plan initiatives are some of the barriers identified by staff which hinder collaboration. 


\section{CONCENTRATING ON EXCELLENCE}

Evaluations from one of the larger IPE programmes $(\mathrm{N}=1852)$ indicate that students value IPE and IDE with $86 \%$ of students rating the sessions as useful and $89 \%$ stating that they felt that working together provided the opportunity to develop skills which would be useful in further employment.

However not all students find the sessions problem free, with particular issues identified as issues: plenary sessions too large (54\%), too much administration (16\%) timing of sessions 'not at the best times' for the cohort involved (32\%) and small groups too dispersed across campus (18\%, reflecting the timetabling challenges for large groupings). These finding indicate that some issues may possibly be addressed by greater use of technology to allow students to engage with each other for shorter more convenient periods or to interact more effectively on line. This in turn opens up the possibility of wider interdisciplinary experiences which may stretch beyond the campus or even the institution. This has proved successful in a project with smaller cohort groups using 'second life' technology to enable students to work internationally with students in Canada and the UK, thereby combining international and interdisciplinary learning in one endeavor (Gurbutt and Gurbutt, 2015) [13] .

One way of pursuing excellence in learning and teaching in interdisciplinary education is to explore flexible, meaningful learning opportunities in work based learning, exploring the adaptation and use of approaches such as Collaborative Learning in Practice (CLIP) (HEE, 2016) [14] which could be used to facilitate IPE in the workplace as well as being a coaching tool. This way of working collaboratively in practice is being trialled on placements. Whilst such approaches are currently focused on face-toface interventions, there is the potential to develop the use of technology in work-based learning. A recent project considered the use of small bespoke flipped classroom sessions which could be accessed flexibly in advance of short interprofessional teaching sessions on placements.

All of these approaches are driven be a shift towards equipping the staff member with technology rather than focusing on providing technology in the teaching environment. Hence the deployment of tablet computers to all academic staff enabling them to prepare, develop, deliver and evaluate sessions in a flexible way using technology with which they can become practised and familiar. Ensuring users have the right technology to embrace fully the digital agenda and parity of device is key to the successful support and integration across the user community. Device provision also encourages and enhances adoption of new and innovative digital approaches, and provides opportunities to develop content and collaborate with colleagues from any location. A unified approach to the provision of devices means that staff can share learning about technology with each other and reinforce each other's learning and experience with using the device.

A diverse range of learning technologies are available to support inclusive learning opportunities. Techsmith Relay Event Capture technology records in-class content allowing for simple sharing through a video catalogue, hosted by the university, to which content is uploaded, for use across academic disciplines. Office Mix, Articulate Presenter and Office Sway provide a broad range of content creation concepts ranging from basic presentation style delivery built on PowerPoint, to fully immersive interactive and engaging online material. This allows academic staff to build variety into their teaching portfolio and select the best tool for particular learning environments.

Standardising assessment methods for digital submission and feedback are also essential elements of successful learning and teaching interventions and can also be used across disciplines. For essaybased submission, the use of Turnitin and Grademark provide the flexibility of online access. Turnitin can be used as a tool to help students to understand the need for careful and appropriate citation. Grademark is a marking tool which enables feedback to be provided in audio files, on text comments, summary comments and grading across a predetermined assessment rubric. Hence the student can access a variety of types of feedback enabling them to make full use of the review of assessed work. The development of transferrable 'Quick Marks' encourage cross-community conversation, feedback developed in one discipline can easily be transposed to another ensuring students receive consistent commentary on their work.

Collaboration is a critical component to support interdisciplinary working and Adobe Connect, OneNote Notebook and Skype offer audio, video, text and image sharing capabilities, ensuring seamless access to developing content across a range of portable and desktop devices. Staff are able to liaise with each other from anywhere on the campus or beyond the campus and edits, recommendations and updates are shared in real time.

The prolific development and availability of web-based tools also offer exciting opportunities to collaborate with colleagues in innovative and flexible ways, developing skills and connections which 
improve the learning experience. Central to this is the sharing of expertise and the recognition that the work of both learning technologist and academic colleagues are enhanced by close collaborations which enable both skill sets to combine to provide engaging, accessible materials. This is one of the ways in which teams 'perform' good teaching.

\section{CREATIVITY AN CHALLENGES}

Returning to the analogy of the theatre 'frontstage and backstage' work, these closer links between learning technologists and academics to co-produce and sometimes co-create learning experiences can enable a broader creativity in the teaching experience and thereby an improved student experience. Although some teaching staff are clearly very able in term of technology use themselves, many find the pace of change in technology for learning and teaching overwhelming and where they may gain competence sometimes still feel that they lack the confidence to fully utilise technology. Staff on CELT courses for initial Learning and Teaching Development cite lack of time as an issue in engaging with technology. Working closely with TELT teams may enable staff to receive the guidance and support they need to trial new approaches and to make connections with other staff in other disciplines who have a shared interest in a particular TEL approach. This has the potential to create an environment in which Communities of Practice (Lave and Wenger, 1991) [15] can emerge and develop. The conversation is being change by the closer alliance of CELT and TELT to demonstrate that the 'team behind teaching' can be much broader than the individuals immediately visible to students. There are challenges inherent in sustaining the momentum of a digital shift. Investment across the institution to support policy is only part of the story, such an initiative needs to be accompanied by a cultural shift. Initiatives which succeed are often as a result of positive staff relationships in which personal connections, mentoring and coaching may play a part. Consolidation is also important with space given to not only introducing new approaches to learning and teaching, but also to sustainability and learning from practice in a continuous cycle of enhancement.

\section{CONCLUSIONS}

There is benefit to the development of close working relationship between the academic teaching community and learning technology teams, often the two are separated by the structures of higher education institutions which arrange services and schools in separate parts of the organisation. Where these divides can be crossed or navigated there are gains for both parts of the organisation. Interdisciplinary education enables a vehicle for not only students, but also staff to learn from each other and work together on shared learning projects which enable cross fertilisation of approaches and knowledge. The barriers which exist in IDE and the challenges of working across discipline and professional boundaries are similar to those experienced by academic and technical staff in making connections, identifying opportunities and recognising resources. The lessons learned from working collaboratively in IDE demonstrate the importance of recognising that the positive experience of the 'audience' is enhanced when all cast members are enabled to work harmoniously in their specialised tasks each supporting the others, and as a result the 'performers' and 'performance' become synonymous.

\section{REFERENCES}

[1] Centre for Advancement of Interprofessional Education, Accessed 161 2017, Retrieved from: www.caipe.org/

[2] E.Goffman, The Presentation of Self in Everyday Life. London: Penguin. 1959.

[3] Dearing Report, National Committee of Inquiry into Higher Education, London: Dept. of Education, 1997.

[4] HEFCE, National Student Survey, 2017, Accessed 161 2017, Retrieved from: http://www.hefce.ac.uk/lt/nss/

[5] NUS/ QAA, Student Experience Research, 2012, Accessed 1612017 , Retrieved from: https://www.nus.org.uk/PageFiles/12238/2012 NUS QAA Independent Learning and Contac $\underline{t \text { Hours.pdf }}$

[6] HEFCE, Research Excellence Framework, 2017, Accessed 161 2017, Retrieved from: http://www.ref.ac.uk/ 
[7] Dept. of Education, Teaching Excellence Framework, 2017, Accessed 161 2017, Retrieved from: https://www.gov.uk/government/news/universities-rated-in-teaching-excellence-framework London: GovUK

[8] V. Butcher, J. Smith, J. Kettle, L. Burton, Review of Good Practice in Employability and Enterprise Development by Centres of Excellence in Teaching and Learning. York: Higher Education Academy, 2011.

[9] GMC, Outcomes for Graduates, 2015, Accessed 1612017 , Retrieved from: https://www.gmcuk.org/Outcomes for graduates Jul 15 1216.pdf 61408029.pdf

[10] E.J.H. Spelt, H.J.A Biemans, H. Tobi, P.A. Luning, M. Mulder, "Teaching and Learning in Interdisciplinary Higher education : A systematic Review”, Educ Psychol Rev, 2009, 21, pp. 365. https://doi.org/10.1007/s10648-009-9113-z

[11] D.J Gurbutt,. R. Gurbutt "Telling Tales - creating a space for stories in practitioner education." In Brewer G. and Hogarth, R. (Eds) Creative Education, Teaching and Learning: Creativity, engagement and the student experience. London: Palgrave Macmillan, 2015.

[12] University of Central Lancashire, Learning and Teaching Strategy: Student Access, Experience and Success. Preston: UCLan, 2017.

[13] R. Gurbutt, D.J.Gurbutt, "Learning in Virtual worlds" in Brewer, G. and Hogarth, R (eds) Creative Education Teaching and Learning: Creativity, engagement and the student experience. London: Palgrave Macmillan, 2015.

[14] Health Education England, Collaborative Learning in Practice. 2016. Accessed 1612017 , Retrieved from: https://www.hee.nhs.uk/sites/default/files/documents/CLiP\%20\%20What\%20it\%20is.pdf

[15] J. Lave, E. Wenger, Situated Learning: Legitimate peripheral participation. Cambridge: Cambridge University Press. 1991 